\title{
ESTIMADORES OBTENIDOS DE LA HIPÓTESIS DE EFICIENCIA ESPECULATIVA EN EL MERCADO CAMBIARIO
}

\author{
Roberto Hernández-Paniagua*
}

Tecnológico de Monterrey, Campus Ciudad de México

(Recibido 18 de febrero 2003, aceptado 19 de septiembre 2003)

\section{Resumen}

Basado en el análisis clásico de los modelos de regresión lineal por el método de mínimos cuadrados ordinarios (OLS), se prueba la eficiencia de los estimadores nuestrales obtenidos de la ecuación de la hipótesis de eficiencia especulativa (SEH), relacionada con la teoría de la Paridad de las Tasas de Interés (IRP) para el periodo de transición democrática del año 2000 en México. Asimismo, se evalúan los sistemas de determinación de los tipos de cambio forward en los mercados cambiarios.

\section{Abstract}

Based on the classic model of lineal regression by the method of ordinary least squares (OLS), the efficiency of the sample estimators obtained from the Speculative Efficiency Hypothesis (SEH), which is related to the Theory of Interest Rate Parity (IRP) during the period of democratic transition in July 2000 in Mexico is proven. Also, we evaluate the systems of determination of the forward exchange rates in the exchange markets.

Clasificación JEL: C12, F31

Palabras clave: Paridad de tasas de interés, Arbitraje internacional

* Tecnológico de Monterrey, Campus Ciudad de México. Calle del Puente 222, Col. Ejidos de Huipulco, Deleg. Tlalpan, México D.F. Teléfono: +52(55)5483 2020 ext. 1305. Correo electrónico: 00969726@academ01.ccm.itesm.mx

El autor desea agradecer el apoyo de la Maestra Esperanza. Sainz López por su invaluable ayuda y sugerencias al presente documento de investigación. 


\section{Introducción}

El motivo por investigar la eficiencia de los estimadores obtenidos de la regresión de la hipótesis de eficiencia especulativa ( $\mathrm{SEH}$ ), a partir de la teoría de la Paridad de las Tasas de Interés (IRP) es que, en fechas cercanas a las elecciones presidenciales del año 2000 en México se pudo apreciar que, a pesar de que las tasas de interés domésticas se incrementeron de manera importante con las políticas restrictivas establecidas por el Banco de México, el tipo de cambio se comportó de una forma impredecible de acuerdo con la Teoría de la Paridad de las Tasas de interés, ya que no sólo mantuvo su cotización frente al dólar, sino que se apreció.

En diversos estudios dentro de la literatura de finanzas internacionales, se menciona la teoría de la Paridad de las Tasas de Interés como una de las relaciones más reconocidas. Esta teoría establece que el tipo de cambio reflejará cambios en su cotización con relación a los diferenciales de tasas de interés de los países involucrados. Sin embargo, en el caso mexicano existe la duda de que si son realmente las tasas de interés las que pueden explicar las cotizaciones de las monedas. En muchas ocasiones, se puede observar que los aspectos de carácter político ocasionan presiones sobre una moneda determinada (Carstens, 1987), también se mencionan problemas de información asimétrica (Werner, 1997) y rebalanceo de portafolios entre los grandes bancos mundiales (Lyons, 1997). Entonces ¿qué se puede decir de la eficiencia de la IRP y sus estimadores para establecer pronósticos del tipo de cambio peso/dólar?

El siguiente documento de trabajo se dividirá en tres partes. En la primera parte, se revisarán los conceptos subyacentes a la teoría de la Paridad de las Tasas de Interés; tales como las condiciones de equilibrio en los mercados de divisas, la Ley de un sólo precio y el arbitraje sobre el proceso de formación de precios spot y forward en los mercados internacionales de divisas y, por último, de la estrecha relación entre estas variables.

En la segunda parte, se enunciará el modelo $S E H$ de regresión lineal, y se probará por el método de mínimos cuadrados ordinarios. Para ello, se analizará la serie de tiempo diaria de la cotización peso/dólar spot durante el periodo del 2 de octubre de 1998 al 14 de noviembre del 2000. De la misma forma y en el mismo periodo, se construirá una serie de tiempo sintética forward utilizando la IRP, la cual se analizará junto con la original para explicar el porqué de las diferencias. En el apartado final, se presentará un cuadro resumen de todas las pruebas realizadas al modelo y se formularán algunas conclusiones y recomendaciones al mismo.

\section{La Paridad de las Tasas de Interés}

La determinación de los tipos de cambio en el futuro viene dada por la teoría de la Paridad de las Tasas de Interés. Los tipos de cambio adelantados (forward exchange rates) y los tipos de cambio al día (spot exchange rates) se encuentran interrelacionados por el nismo tipo de arbitraje que regula la Ley de un sólo precio. ${ }^{1}$

1 Esta Ley se mantiene cuando un activo contingente tiene el mismo valor bajo cualquier estrategia de inversión que lo replique. Véase Pliska (2001). 
La Ley de un sólo precio es válida para un modelo de mercado de divisas si no existen dos estrategias de inversión, que en el tiempo de su realización $(t+1)$ tengan un rendimiento igual, pero que en el tiempo de su formación $(t)$ el costo sea diferente, lo anterior puede ser expresado de la siguiente forma :

"Se viola la ley de un sólo precio cuando existen dos estrategias de inversión $H_{1}$ y $H_{2}$ tal que el valor del portafolio de divisas en el tiempo $t+1$ sea $V_{1}(w)=$ $V_{2}(w)$, para todo $w \in \Omega$, pero $V_{1} \neq V_{2}$ en el tiempo $t . "$

Por otra parte, la Teoría de la Paridad de las Tasas de Interés implica que si las tasas de interés domésticas son mayores que las tasas de interés foráneas, la moneda foránea se estaría vendiendo a premio en el mercado forward. De manera contraria, si las tasas de interés domésticas fueran menores que las tasas de interés foráneas, la moneda foránea se estaría vendiendo a descuento. ${ }^{2}$

El movimiento de fondos entre dos divisas con el fin de tomar ventaja de los diferenciales entre las tasas de interés, es también un factor determinante del spread entre las tasas spot y forward; de hecho, el premio o descuento forward está íntimamente relacionado al diferencial entre las tasas de interés. En un mercado eficiente sin costos de transacción, el diferencial de tasas de interés deberá ser aproximadamente igual al diferencial en el tipo de cambio forward. Cuando esta condición se cumple, se dice que la tasa forward está en paridad de interés y el mercado se encuentra en equilibrio.

La Paridad de las Tasas de Interés asegura, que el retorno de una posición cubierta en inversión en moneda foránea sólo igualará la tasa de interés doméstica sobre una inversión con idéntico riesgo. De tal forma, se eliminará la posibilidad de tener una "máquina de hacer dinero". Cuando esta condición se cumple, el diferencial cubierto de tasas de interés es cero. Si esto no se cumple, existen oportunidades de arbitraje al mover dinero de un mercado a otro. ${ }^{3}$ Cuando la Ley de un sólo precio falla, existen oportunidades de arbitraje, estas oportunidades de arbitraje pueden ser definidas como una estrategia $H$ tal que: $V_{0}=0, V_{1} \geq 0$ y $\mathrm{E}\left[V_{1}\right]>0$. Lo anterior, es equivalente a decir, que existe una probabilidad de obtener algún rendimiento positivo en el portafolio de divisas con un costo inicial de cero.

Regresando a la Teoría de la Paridad de las Tasas de Interés, ésta es frecuentemente aproximada con la siguiente ecuación: ${ }^{4}$

$$
F(t, T)=S(t) \frac{1+i_{d}(T / 360)}{1+i_{f}(T / 360)},
$$

donde $i_{d}$ es la tasa de interés doméstica, $i_{f}$ es la tasa de interés foránea, $F(t, T)$ es el tipo de cambio forward en el tiempo $T$ y $S(t)$ es el tipo de cambio spot.

2 Véase Ross, Westerfield and Jaffe (1996, pág.831) para una explicación más detallada.

3 Estas oportunidades son generalmente de corta duración, ya que al existir un desequilibrio en el mercado, las mismas condiciones de oferta y demanda harán que estas oportunidades desaparezcan en el corto plazo.

4 Es muy frecuente encontrar en libros o artículos de finanzas internacionales esta relación cxpresada en términos de tiempo continuo. 
La Teoría de la Paridad de las Tasas de Interés es una de las mejores relaciones en finanzas internacionales. De hecho, en los mercados de eurodivisas, la tasa forward es calculada a partir de los diferenciales entre las dos monedas involucradas, utilizando las restricciones de no-arbitraje. Las desviaciones a partir de la paridad de las tasas de interés ocurren entre los mercados de capitales nacionales. Sin embargo, estas desviaciones tienden usualmente a ser pequeñas y de corto plazo, siempre y cuando se mantenga el control de capitales, la imposición de impuestos sobre pago de intereses a extranjeros y los costos de transacción.

El periodo de análisis que se eligió para la presente investigación comprende desde el 2 de octubre de 1998 al 14 de noviembre del 2000. Se eligió este periodo en virtud de que fue durante fechas posteriores a la elección del 2 de julio del 2000, que se observó en el mercado cambiario una apreciación del peso con respecto al dólar, lo cual era impredecible de conformidad con el comportamiento de las tasas de interés en ambos países y, por ende, con la IRP y los tipos de cambio forward.

Se consideró que para probar la eficiencia de la IRP en el corto plazo, los datos deberian ser de alta frecuencia. ${ }^{5}$ Es por ello, que se tomaron datos diarios de la cotización de cierre del tipo de cambio spot y de las tasas de interés de Cetes y Libor overnight a un día. De acuerdo con la fórmula de paridad de tasas de interés, se esperaría que si la tasa de interés doméstica aumentara por encima de la tasa de interés foránea, el tipo de cambio forward subiría de precio.

Se puede apreciar claramente en la gráfica 1, cómo a partir del mes de julio del 2000 existe un comportamiento estable del tipo de cambio, incluso con tendencia hacia la baja durante los meses de agosto y septiembre, para luego tener un pequeño repunte hacia el final del año. De igual forma, se observa en la gráfica 2 , cómo se comportaron las tasas de interés de ambos países. ${ }^{6}$

En la gráfica 2, las tasas de interés de Estados Unidos se mantuvieron relativamente constantes durante el periodo de análisis, lo cual significó que el comportamiento de la tasa de interés en México debería ser muy similar al del tipo de cambio spot; lo anterior, parece cumplirse al inicio de la gráfica; sin embargo, para los periodos subsecuentes no es así del todo.

La relación existente entre las tasas de interés de ambos países tiende a aproximarse con el tiempo, en virtud de las metas inflacionarias de ambos gobiernos con respecto a diferentes acuerdos binacionales y con diferentes organismos internacionales. ${ }^{7}$ Además, recordemos que la IRP está muy relacionada con el diferencial inflacionario por medio de la paridad de poder de compra; de hecho, la teoría de la paridad de poder de compra puede ser vista como una teoría sustituta de la IRP, pero aplicable en un horizonte de largo plazo (Castro y Ortíz, 1996).

5 Se consideran datos de alta frecuencia aquellos que son de muy corto plazo (un día, una hora o inclusive por minutos).

${ }^{6}$ Se utilizaron tasas de interés de Cetes a un día y la Libor overnight por ser las tasas líderes del mercado de dinero en ambos países.

7 Acuerdos comerciales como NAFTA (TLCAN), y acuerdos con organismos internacionales tales como el FMI, Banco Mundial y BID. 

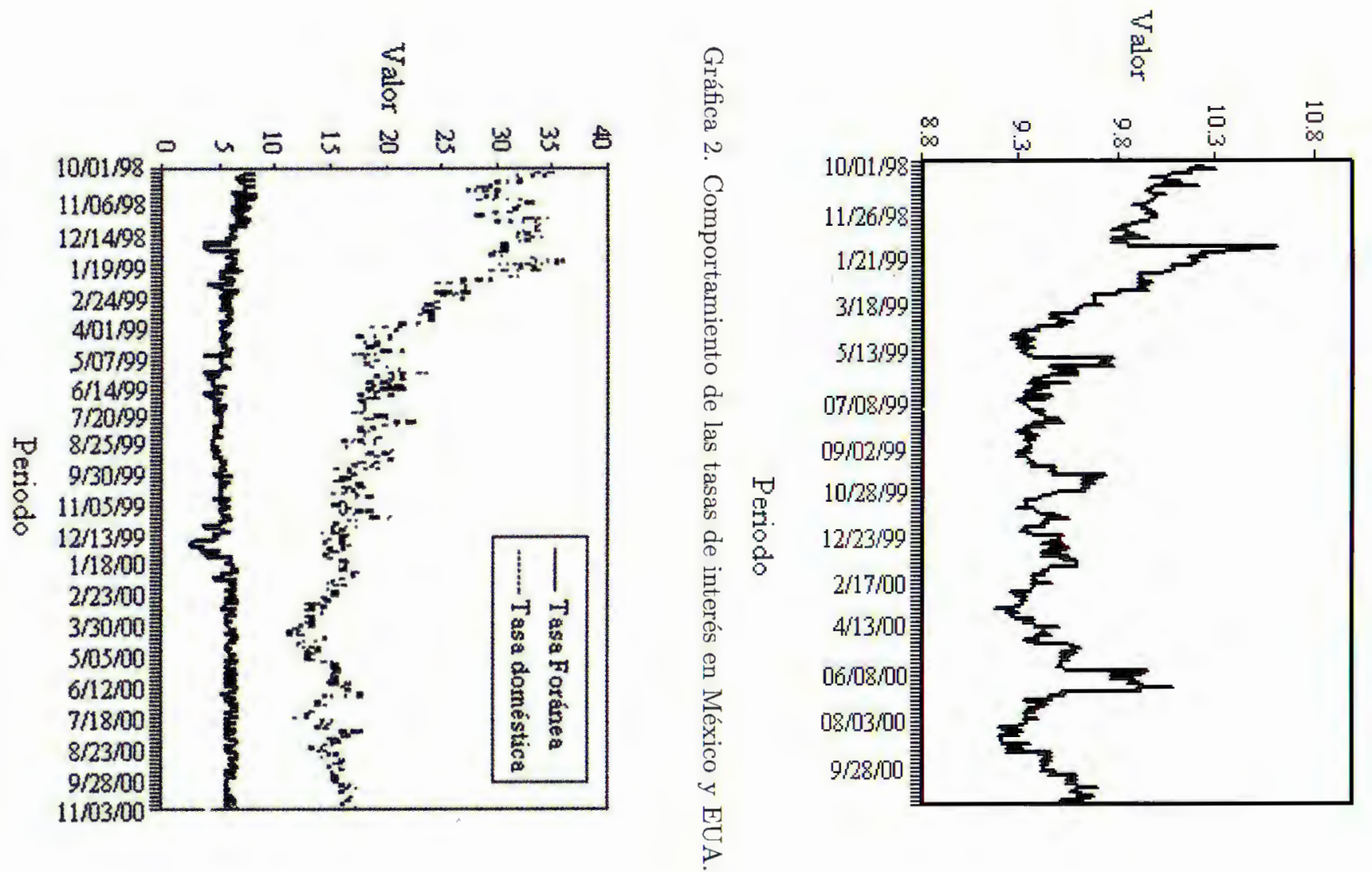
Aplicando la fórmula de la IRP (1) y estableciendo el periodo a un día, se puede observar en la gráfica 3 el comportamiento del tipo de cambio forward sintético durante el periodo de análisis, seleccionado conjuntamente con el tipo de cambio spot.

Gráfica 3. Comportamiento del tipo de cambio spot y forward Sintético.

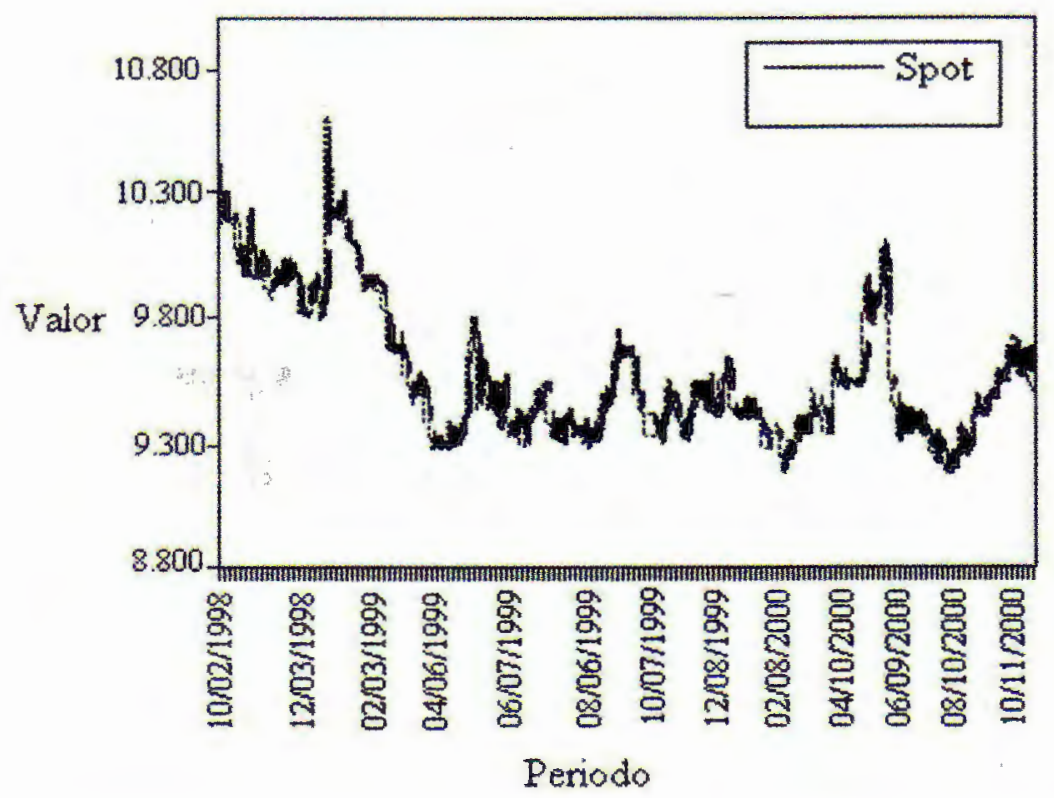

La primera pregunta obligada sería ¿se parecen las gráficas 1 y 3 ? La respuesta es que a primera instancia sí, pero existe otra pregunta ¿Qué tan semejantes? Esto se validará con la realización de pruebas empíricas. Éstas consisten en la formulación de un modelo de regresión lineal por el método de mínimos cuadrados ordinarios, teniendo como variable dependiente el tipo de cambio spot, $S(t)$, y como variable independiente el tipo de cambio forward , $F(t, T)$, el cual involucra implícitamente los niveles de tasas de interés tanto en México como en los Estados Unidos por medio de la paridad de las tasas de interés, como se mencionó en la fórmula (1).

Sin embargo, para poder aplicar el método de regresión lineal por mínimos cuadrados ordinarios, se deben hacer algunas pruebas para verificar la existencia de una relación de tipo lineal entre las variables; en caso de que no existiera, será necesario efectuar transformaciones para obtener la relación buscada o utilizar un modelo de regresión no lineal (cuadrático o de otro orden superior). ${ }^{8}$

8 La transformación logarítmica es frecuentemente utilizada en el análisis de series de tiempo, en virtud de que permite analizar las diferencias suavizando los movimientos bruscos en la serie original. 
Un método sencillo y frecuentemente utilizado es graficar la relación entre las variables y observar una probable existencia de un comportamiento lineal, una vez que se ha comprobado lo anterior, se puede proseguir con las pruebas. La relación del tipo de cambio spot y el tipo de cambio forward se presenta en la gráfica 4.

Evidentemente, la relación entre estas dos variables es lineal y no es necesario realizar transformación alguna, por tal motivo, se puede concluir que es factible la utilización de un método de regresión lineal para poder estimar que tan eficiente es en el corto plazo la IRP y el tipo de cambio forward sintético.

Gráfica 4. Tipo de cambio spot vs forward.

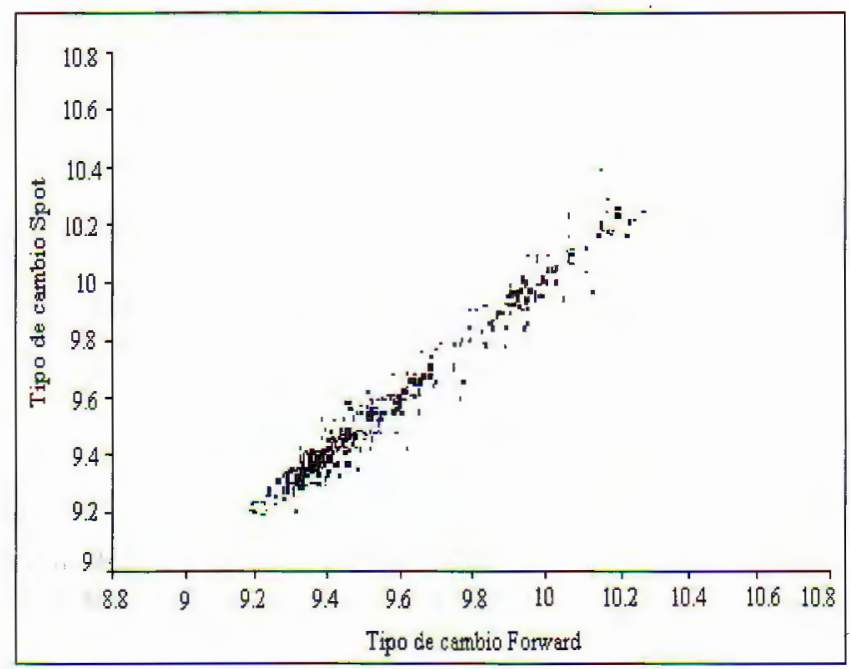

Sin embargo, en la misma gráfica se puede apreciar la existencia de algunas observaciones que no caen dentro de la tendencia lineal. Estas observaciones, tal vez puedan ocasionar algunos problemas no deseados dentro de los resultados del modelo.

Por tratarse de un análisis de datos en series de tiempo, se sugiere la verificación de la no-estacionariedad de la serie. Por tal motivo, se correrá en primera instancia una prueba de raíces unitarias para verificar la estacionariedad de la media y posteriormente una prueba de homoscedasticidad para verificar los segundos momentos.

Recordando un poco la teoría detrás de una prueba de raíces unitarias, pensemos en un modelo $A R(1)$ representado por medio de la siguiente ecuación:

$$
S_{t}=\mu+\beta_{1} S_{t-1}+\epsilon_{t},
$$

donde si el valor de $\beta_{1}$ es estadísticamente igual a 1 , estaríamos ante lo que comúnmente se denomina una caminata aleatoria con tendencia, (random walk with a drift) 
De esta forma, la existencia de una raíz unitaria garantiza que todos los incrementos sobre el drift serán un ruido blanco puro $\mathrm{y}$, por lo tanto, la serie no es estacionaria en la media. La prueba de hipótesis se establece de manera formal como: $H_{0}: \beta_{1}=1$ (Existe una raíz unitaria) y $H_{1}: \beta_{1} \neq 1$ (No existe una raíz unitaria). Se usa la prueba Dickey-Fuller y los resultados son los siguientes:

Cuadro 1. Prueba de raíces unitarias para la serie de tipo de cambio spot.

\begin{tabular}{|c|c|c|c|c|}
\hline Prueba ADF & -15.9050 & $1 \%$ Valor crítico* & \multicolumn{2}{|c|}{-3.4446} \\
\hline & & $5 \%$ Valor crítico & \multicolumn{2}{|c|}{-2.8671} \\
\hline & & $10 \%$ Valor crítico & \multicolumn{2}{|c|}{-2.5697} \\
\hline \\
\hline Variable & Coef. & Error típico & Estad.-t & Prob. \\
\hline $\mathrm{D}(\operatorname{SPOT}(-1))$ & -0.91142 & 0.05730 & -15.9050 & 0.0000 \\
\hline $\mathrm{D}(\operatorname{SPOT}(-1), 2)$ & 0.01529 & 0.04271 & 0.35816 & 0.7204 \\
\hline $\mathrm{C}$ & -0.00113 & 0.00219 & -0.51800 & 0.6047 \\
\hline$R^{2}$ & 0.449094 & Media Var. Dep. & \multicolumn{2}{|c|}{$4.00 \mathrm{E}-05$} \\
\hline$R^{2}-a j u s t a d a$ & 0.447080 & D.E. Var. Dep. & \multicolumn{2}{|c|}{0.069126} \\
\hline Error típico de regresión & 0.051401 & Criterio Akaike & \multicolumn{2}{|c|}{-3.092884} \\
\hline Suma resid. cuadrado & 1.445200 & Criterio Schwarz & \multicolumn{2}{|c|}{-3.069376} \\
\hline Verosimilitud Log & 853.5432 & Estad.-F & \multicolumn{2}{|c|}{222.9550} \\
\hline Estad. Durbin-Watson & 1.998038 & Prob(Estad.-F) & \multicolumn{2}{|c|}{0.000000} \\
\hline
\end{tabular}

* Valores críticos de MacKinnon para rechazar la hipótesis de raíz unitaria. Variable dependiente: D(SPOT,2). Muestra ajustada: 10/02/1998 a 11/14/2000. Observaciones: 550 .

En virtud de que el estadístico $t$ es menor (cae del lado izquierdo) que el valor crítico, se rechaza la hipótesis nula de la existencia de una raíz unitaria y se concluye que la serie de tiempo del tipo de cambio spot, $S(t)$, es estacionaria en la media.

\section{El Modelo SEH}

En el caso que nos ocupa la ecuación de regresión que se correrá será la siguiente:

$$
S(t+T)=\beta_{0}+\beta_{1} F(t, T)+\epsilon(t+T) .
$$

Esta ecuación se relaciona con la ecuación (1) debido a que la tasa spot en el tiempo $(t+T)$ debería ser aproximadamente igual a la tasa forward en $(t, T)$. En la ecuación (3), los valores de $\beta_{0}$ y $\beta_{1}$ deberían ser igual a cero y uno respectivamente, para que $F(t, T)$ sea eficiente. Esta ecuación es conocida como la hipótesis de eficiencia especulativa $(S E H) .^{9}$

\footnotetext{
9 Esta ecuación establece que el premio forward es un pronóstico insesgado de la apreciación del tipo de cambio.
} 
Una vez que se obtienen los parámetros, se verificará que se cumplan las siguientes siete supuestos típicos acerca de las regresiones lineales.

1. Método bien especificado con $\beta^{\prime}$ s constantes y lineales en los parámetros, ${ }^{10}$

2. El valor esperado del error es igual a cero,

3. La varianza del error debe ser igual a $\sigma^{2}$,

4. La covarianza de los errores debe ser igual a cero,

5. Las $X^{\prime} s$ no son estocásticas, por lo tanto, no puede existir correlación entre las variables independientes y los errores,

6. No existe Multicolinealidad; es decir, las columnas de la matriz son linealmente independientes,

7. Prueba de normalidad: Los errores deben distribuirse normalmente. ${ }^{11}$

Corriendo la regresión de acuerdo con la fórmula (3), se obtienen los siguientes resultados:

Cuadro 2. Estadísticas de la regresión del modelo $S E H$.

\begin{tabular}{|c|c|c|c|c|}
\hline \multicolumn{5}{|c|}{ MCO // Variable Dependiente es SPOT } \\
\hline \multicolumn{5}{|c|}{ Ouestra: 10/02/1998 11/14/2000 } \\
\hline \multicolumn{5}{|c|}{ Obs. incluidas: 553 } \\
\hline Variable & Coef. & Error típico & Estad.-t & Prob. \\
\hline \multicolumn{5}{|c|}{} \\
\hline FWD & 0.96878 & 0.008019 & 120.8183 & 0.0000 \\
\hline C & 0.29347 & 0.076777 & 3.822389 & 0.0001 \\
\hline$R^{2}$ & 0.96362 & Media Var. Dep. & 9.565665 \\
\hline$R^{2}$ ajustada & 0.96356 & D.E. Var. Dep. & 0.272081 \\
\hline Error típico de regresión & 0.05194 & Criterio Akaike & -5.911779 \\
\hline Suma resid. cuadrado & 1.48638 & Criterio Schwarz & -5.896172 \\
\hline Verosimilitud Log & 851.934 & Estad.-F & 14597.07 \\
\hline Estad. Durbin-Watson & 1.77777 & Prob(Estad.-F) & 0.000000 \\
\hline
\end{tabular}

Considerando un nivel de significancia de $.05(\alpha=.05)$, se plantea la hipótesis nula y alternativa de la siguiente forma; ${ }^{12}$

$$
\begin{gathered}
H_{0}=\beta_{1}=0 \text { (La variable exógena no tiene sentido). } \\
H_{t}=\beta_{1} \neq 0(\text { La variable exógena explica a la endógena }) .
\end{gathered}
$$

10 Esta suposición es frecuentemente llamada como "el modelo correcto".

11 Esta condición es importante para la distribución de los estimadores $\hat{\beta}$.

12 El nivel de significancia es la probabilidad de cometer el error tipo I; es decir, rechazar la hipótesis nula $\left(H_{0}\right)$ cuando es verdadera. 
El coeficiente de determinación o $R^{2}$ pareciera ser adecuado ya que establece que un $96.36 \%$ de los movimientos del tipo de cambio spot son explicados por el tipo de cambio forward, que se encuentra relacionado con la teoría de la IRP, como se explicó detalladamente en el capítulo 1 de la presente investigación. ${ }^{13}$

Si los $P$-Values son menores que las $F \alpha^{\prime} s$, entonces rechazamos la hipótesis nula. ${ }^{14}$ De esta forma, la conclusión que se obtiene es que con un nivel de significancia de .05 , la variable exógena explica el comportamiento del tipo de cambio spot. Sin embargo, con un nivel de confianza de $95 \%$, la $S E H$ no se cumple para este periodo, ya que los coeficientes buscados $\beta_{0}$ y $\beta_{1}$ son estadísticamente diferentes de 0 y 1 respectivamente. A continuación, se iniciará el análisis de los siete supuestos básicos del modelo de regresión lineal.

\subsection{Supuesto 1: Coeficientes constantes}

Observemos ahora que pasa con la estructura de la serie; es decir, se tratará de probar que las $\beta$ 's son constantes en el tiempo. Esto se hará corriendo la prueba de Chow Breakpoint test y analizando el estadístico. ${ }^{15}$ Se tomarán dos periodos, el primer periodo será desde el 2 de octubre de 1998 al 31 de mayo del 2000, y el segundo periodo será del 1 de Junio del 2000 al 14 de noviembre del 2000. Las hipótesis nula y alternativa son las siguientes:

$H_{0}=\mathrm{La} \beta_{1}$ es constante durante todo el periodo (no hay cambios estructurales en el modelo),

$H_{1}=$ La $\beta_{1}$ no es constante durante todo el periodo (existen cambios estructurales en el modelo).

Los resultados obtenidos de la utilización del Chow Breakpoint test son los siguientes:

Cuadro 3. Prueba de Chow.

\begin{tabular}{|c|c|c|c|}
\hline \multicolumn{4}{|c|}{ Chow Breakpoint Test: $5 / 31 / 2000$} \\
\hline F-statistic & 0.050417 & Probability & 0.950837 \\
\hline Log likelihood ratio & 0.101560 & Probability & 0.950488 \\
\hline
\end{tabular}

Ya que el estadístico $F$ toma un valor muy pequeño y por consiguiente el $P$ value es muy alto (mayor que el $\alpha=.05$ ), no se rechaza la hipótesis nula. Por

13 A pesar de ser un estadístico importante, una $R^{2}$ no necesariamente es mala cuando ésta se encuentra por niveles bajos, digamos un $30 \%$, ya que si el estadístico $F$ es significativo, entonces se considera que la regresión y las variables independientes son buenas en su conjunto.

14 En el programa económetrico e-views no es necesario realizar pruebas de hipótesis utilizando las tablas de distribución, lo que se hace es observar los Prob (Probability value) que aparecen en las estadísticas de la ecuación de regresión y compararlos contra el porcentaje del error tipo I, si el Prob es menor que el $\alpha$, entonces se rechaza la hipótesis nula y viceversa.

15 La idea de la prueba de Chow es ajustar la ecuación de regresión inicial en dos o más sub-muestras y poder observar si existen diferencias significativas en las ecuaciones estimadas para cada periodo. Una diferencia significativa indica un cambio estructural en la relación de las variables a ser analizadas. 
lo tanto, se puede concluir que los coeficientes del modelo son estables en el tiempo.

\subsection{Supuesto 2: Errores con media cero}

Ahora observemos las estadísticas e histograma de los residuales en la gráfica 5 . Se puede apreciar la forma simétrica de la distribución, verificando los valores estadísticos de dicha gráfica se puede apreciar que el valor de la media es un número muy pequeño (9.04 E-17), y que la desviación típica es un número relativamente grande (.051891), con lo cual se puede concluir que con un nivel de significancia de .05 , no se rechaza la hipótesis nula de que el valor de los errores es cero. Con lo cual se prueba el supuesto número $2 .{ }^{16}$ La prueba de hipótesis formal sería de la siguiente:

$$
\begin{gathered}
H_{0}=\mu_{\epsilon}=0 \text { (La media de error es igual a cero), } \\
H_{i}=\mu_{\epsilon} \neq 0 \text { (La media del error es diferente de cero). }
\end{gathered}
$$

\subsection{Supuesto 7: Distribución normal de los errores}

El supuesto siete, referente a la normalidad de la distribución de los errores se puede deducir observando el estadístico Jarque-Bera, el cual es básicamente una prueba de simetría y curtosis de la distribución. ${ }^{17}$

También se puede apreciar en la gráfica 5, que la forma de la distribución de los errores es simétrica pero degenerada de manera leptocurtica. ${ }^{18}$ En virtud de que el $P$-Value de $J B$ es menor que .05 , se rechaza la hipótesis nula de que los errores se distribuyen de manera normal.

\subsection{Supuesto 3: Varianza constante}

Se analizará ahora el supuesto 3 referente a la varianza constante, realizando pruebas de heteroscedasticidad. Se realizará la prueba de White y las hipótesis son las siguientes:

16 Cuando las muestras son grandes, es condición suficiente la forma simétrica de la distribución de los residuales para establecer que los estimadores se distribuyen de manera normal por el teorema del límite central. Mendenhall, Wackerly y Sheaffer (1994, pág. 296).

17 Esta prueba debería en realidad llamarse Bowman-Shelton-Jarque-Bera, ya que Bowman y Shelton fueron los primeros en desarrollar el modelo (1975), mientras que Jarque y Bera culminaron el trabajo en (1979). El estadístico se construye de la siguiente manera : $J B=$ $n\left[\frac{A^{2}}{6}+\frac{(K-3)}{24}\right]$, donde $A$ representa el coeficiente de asimetría y $K$ la curtosis, dado que para una distribución normal, el valor de la asimetría es cero y el valor de la curtosis es 3 , en $(K-3)$ se representa un excedente de Curtosis. Bajo la hipótesis nula de que los residuales están normalmente distribuidos, Jarque y Bera demostraron que asintóticamente el estadístico $J B$ sigue una distribución Ji-Cuadrada con dos grados de libertad, por lo tanto, si el valor $p$ del estadístico Ji-Cuadrada calculado en una aplicación es suficientemente pequeño, entonces se puede rechazar la hipótesis de que los errores se encuentran normalmente distribuidos. Pero si el valor $p$ es razonablemente alto, entonces no se rechaza el supuesto de normalidad de los errores.

18 Forma de distribución caracterizada por colas delgadas y forma afilada. 


$$
\begin{gathered}
H_{0}: \text { Hayhomoscedasticidad (varianzaconstante), } \\
H_{I}: \text { Hayheteroscedasticidad (varianzanoconstante). }
\end{gathered}
$$

Gráfica 5. Estadísticas de residuales.

\begin{tabular}{|c|c|}
\hline \multicolumn{2}{|c|}{$\begin{array}{l}\text { Series: Residuals } \\
\text { Sample 101021998 } 11 / 1412000 \\
\text { Coservations } 553\end{array}$} \\
\hline Wean & $9.04 E-17$ \\
\hline Median & $=0.001738$ \\
\hline Maximum & 0.385159 \\
\hline Minimum & -0.365285 \\
\hline Std. Dey. & 0.051891 \\
\hline Skewness & -0.078534 \\
\hline Kutosis & 16.07836 \\
\hline Jarque-Bera & 3941,698 \\
\hline Probability & 0.000000 \\
\hline
\end{tabular}

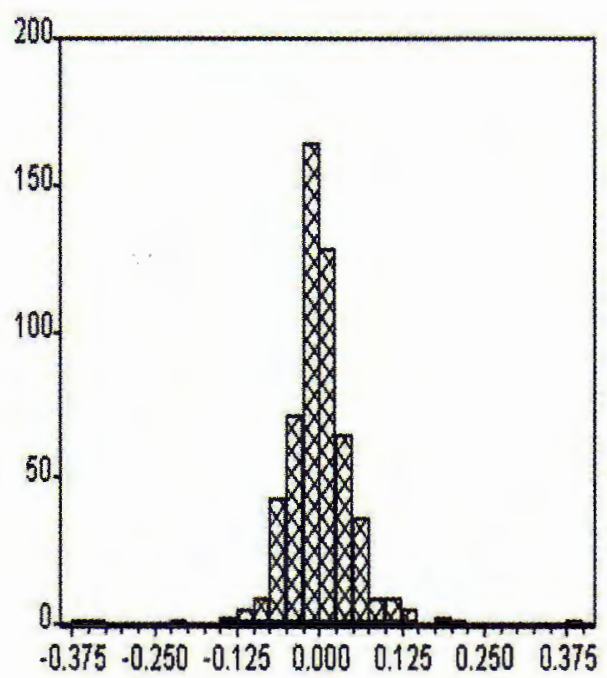

Las estadísticas se pueden apreciar en la cuadro 4. En virtud de que el $P$-Value es menor que el $\alpha=.05$ se rechaza la hipótesis nula. Esto quiere decir que la serie de tiempo tiene varianza no constante. De conformidad con la teoría de series de tiempo, cuando una serie tiene varianza no constante se sugiere el uso de modelos $A R C H$ y $G A R C H$. Estos modelos de serie de tiempo son utilizados cuando existe un problema de heterocedasticidad.

En el documento de investigación número 9701 del Banco de México, realizado por Alejandro Werner, se profundiza acerca de la volatilidad del peso con respecto al dólar y se sugiere el uso de un modelo $G A R C H$ debido a varios factores, entre los cuales destacan los siguientes:

- Cambios importantes en las variables fundamentales provocan una alta incertidumbre sobre los cambios en el futuro.

- La existencia de un problema de información asimétrica entre los participantes del mercado cambiario. ${ }^{19}$

- Los lunes, el tipo de cambio se deprecia en menor medida que durante los demás días de la semana.

- La volatilidad observada durante los días viernes es menor a la observada durante los demás días de la semana.

\footnotetext{
19 Esta situación aparece cuando en un contrato celebrado una de las partes tiene más y mejor información acerca de la otra u otras partes, por lo cual esto lo lleva a tomar mejores decisiones y sacar ventaja en virtud de ello.
} 
Cuadro 4. Estadísticas de prueba de varianza.

\begin{tabular}{|c|c|c|c|c|}
\hline \multicolumn{5}{|c|}{ White Heteroskedasticity Test: } \\
\hline Estad.-F & \multicolumn{2}{|c|}{37.911882} & Prob. & 0.0000 \\
\hline $\mathrm{Obs}^{*} R^{2}$ & \multicolumn{2}{|c|}{67.00056} & Prob. & 0.0000 \\
\hline \multicolumn{5}{|c|}{ MCO // Variable Dependiente $R E S I D^{2}$} \\
\hline Variable & Coef. & Error típico & Estad.-t & Prob. \\
\hline $\mathrm{C}$ & 3.127750 & 0.493574 & 6.336946 & 0.0000 \\
\hline FWD & -0.652860 & 0.101731 & -6.417515 & 0.0000 \\
\hline$F W D^{2}$ & 0.034069 & 0.005238 & 6.504517 & 0.0000 \\
\hline$R^{2}$ & 0.121158 & Media Var. Depend. & 0.002 & \\
\hline$R^{2}$ ajustada & 0.117963 & D.E. Var. Depend. & 0.010 & \\
\hline Error típico regresión & 0.009821 & Criterio Akaike & -9.24 & \\
\hline Suma resid. cuadrado & 0.053044 & Criterio Schwarz & -9.21 & \\
\hline Verosimilitud Log & 1773.501 & Estad.-F & 37.91 & \\
\hline Estad. Durbin-Watson & 1.779684 & Prob(Estad.-F) & 0.000 & \\
\hline
\end{tabular}

\subsection{Supuesto 4: Autocorrelación}

El concepto de no autocorrelación se refiere a que no debe existir correlación entre los errores de diferentes periodos, esto puede ser expresado de la siguiente manera: $\operatorname{COV}\left(\epsilon_{t}, \epsilon_{t-k}\right)=0$, donde $k=1,2, \ldots, K$.

Se analizará este supuesto verificando la prueba Durbin-Watson. ${ }^{20}$ De la primera ecuación que se corrió por mínimos cuadrados ordinarios, se obtuvo un estadístico Durbin-Watson de 1.777 , lo cual a simple vista pudiera parecer que es cercano a dos y que por ello no existe correlación. ${ }^{21}$

Para verificar que tan cerca está el indicador de dos y pensar que efectivamente no hay autocorrelación, se debe conocer la región de rechazo por medio de tablas. Para ello, se usará la tabla 7 del Libro de Greene (1999) y se establecerán los valores críticos de conformidad con los siguientes parámetros: $n=550$

20 El estadístico Durbin-Watson mide la asociación entre los residuales adyacentes. Con regresiones de series de tiempo, la correlación entre los residuales, los cuales son adyacentes en el tiempo, son frecuentemente un problema. La prueba $D W$ es una prueba formal de correlación serial. Si no existe algún problema de asociación entre residuales el valor del estadístico deberá ser cercano a dos.

21 El estadístico está representado por medio de la siguiente fórmula:

$$
D W=\sum_{t=2}^{n} \frac{\left(e_{t}-e_{t-1}\right)^{2}}{\sum e^{2}} \approx 2(1-r) .
$$


(Tamaño de la muestra), $k=1$ (Número de variables explicativas) y $\alpha=.05$ (probabilidad de cometer el error tipo I). Las hipótesis nula y alternativa para la prueba de Autocorrelación son las siguientes:

$$
\begin{gathered}
H_{0} \text { : No hay Autocorrelación, } \\
H_{1} \text { : Si hay Autocorrelación. }
\end{gathered}
$$

El rango de aceptación con la $n$ más grande encontrado en la tabla del libro de Greene (1999) es de $(1.758,2.211)$, con lo cual no se rechaza la hipótesis nula y se puede afirmar que no existe un problema de autocorrelación entre los errores. Sin embargo, dada la restricción que se obtiene por el uso de las tablas, con un número de observaciones limitado $(\mathrm{n}=200)$, se realizará de manera complementaria otra prueba de autocorrelación llamada $L M$ autocorrelation Test o también llamada prueba de Breusch-Godfrey que se hace a través del uso de multiplicadores de Lagrange. Esto consistente en tomar tiempos de retraso que se ajusten con el modelo. ${ }^{22}$ Las hipótesis nula y alternativa son las mismas y los estadísticos que arroja esta prueba con un retraso de uno y dos tiempos, son los que se presentan de manera puntual en la cuadro 5.

\begin{tabular}{|c|c|c|c|c|}
\hline \multicolumn{5}{|c|}{ Breusch-Godfrey Serial Correlation LM Test: } \\
\hline Estad.-F & \multicolumn{2}{|c|}{$\begin{array}{l}\text { ch-Godfrey Serial Correlation LM } \\
2.690828\end{array}$} & Prob. & 0.068719 \\
\hline $\mathrm{Obs}^{*} R^{2}$ & \multicolumn{2}{|l|}{5.368243} & Prob. & 0.068281 \\
\hline \multicolumn{5}{|c|}{ MCO // Variable Dependiente $R E S I D^{2}$} \\
\hline \multicolumn{5}{|c|}{ Test Equation: } \\
\hline \multicolumn{5}{|c|}{ Variable Dependiente: RESID } \\
\hline Variable & Coef. & Error típico & Estad.-t & Prob. \\
\hline FWD & -0.003697 & 0.008288 & -0.446109 & 0.6557 \\
\hline $\mathrm{C}$ & 0.035385 & 0.079352 & 0.445921 & 0.6558 \\
\hline $\operatorname{RESID}(-1)$ & 0.100024 & 0.043329 & 2.308492 & 0.0213 \\
\hline$R E S I D(-2)$ & 0.003840 & 0.043504 & 0.088265 & 0.9297 \\
\hline$R^{2}$ & 0.009707 & Media Var: Depend. & \multicolumn{2}{|c|}{$-4.50 E-15$} \\
\hline$R^{2}$ ajustada & 0.004296 & D.E. Var. Depend. & \multicolumn{2}{|c|}{0.051901} \\
\hline Error típico regresión & 0.051790 & Criterio Akaike & \multicolumn{2}{|c|}{-3.076038} \\
\hline Suma resid. cuadrado & 1.472522 & Criterio Schwarz & \multicolumn{2}{|c|}{-3.044824} \\
\hline Verosimilitud Log & 854.5245 & Estad.-F & \multicolumn{2}{|c|}{1.793885} \\
\hline Estad. Durbin-Watson & 1.970640 & Prob(Estad.-F) & \multicolumn{2}{|c|}{0.147236} \\
\hline
\end{tabular}

Cuadro 5. Estadísticas de pruebas de autocorrelación.

22 La correlación serial del la prueba $L M$ es una alternativa para la prueba de correlación serial general. Para muestras grandes se usa la prueba de Breusch-Godfrey, esta prueba es aplicable si los errores siguen un proceso $A R(p) \circ M A(q)$. Para usar esta prueba se necesita especificar el orden de $p$. 
Se puede apreciar que, en virtud de que el valor del $P$-Value es mayor a $\alpha==.05$, no se rechaza la hipótesis nula, es decir, no existe el problema de Autocorrelación cuando los errores siguen un proceso $A R(1)$ y $A R(2)$.

\subsection{Supuesto 5: $\operatorname{COV}\left[X_{i}, \epsilon_{i}\right]=0$}

A continuación, se probará la condición más importante de la teoría de regresión y ésta es saber si las variables independientes son estocásticas o si no lo son, $\operatorname{COV}\left[X_{i}, \epsilon_{i}\right]=0$. En caso de que lo anterior no se cumpla, los estimadores no solo son sesgados sino también inconsistentes, por ello la regresión que obtengamos no es útil. ${ }^{23}$ El resultado de calcular la covarianza de los residuales con la variable independiente es un valor muy cercano a cero, con lo cual se estaría probando el supuesto número 5.

$$
\operatorname{COV}\left[F(t, T), \epsilon_{i}\right]=0.000000000358
$$

Sin embargo, nuevamente llegamos a la pregunta clásica en estadística ¿Qué tan diferente es esta cantidad de cero? Lo anterior, se puede formalizar corriendo la siguiente ecuación de regresión y observando sus estadísticas, las cuales se presentan en el cuadro 6.

$$
F(t, T)=\beta_{0}+\beta_{1} \epsilon_{i}+\epsilon(t, T) .
$$

\begin{tabular}{|c|c|c|c|c|}
\hline \multicolumn{5}{|c|}{ Variable Dependiente: FWD } \\
\hline \multicolumn{5}{|c|}{ Método: Mínimos Cuadrados } \\
\hline \multicolumn{5}{|c|}{ Muestra: $10 / 02 / 1998$ y $11 / 14 / 2000$} \\
\hline \multicolumn{5}{|c|}{ Observaciones incluídas: 553} \\
\hline Variable & Coef. & Error típico & Estad.-t & Prob. \\
\hline RES & $1.99 \mathrm{E}-11$ & 0.226308 & $8.79 \mathrm{E}-11$ & 1.0000 \\
\hline $\mathrm{C}$ & 9.570971 & 0.011735 & 815.5857 & 0.0000 \\
\hline$R^{2}$ & 0.000000 & Media Var. Depend. & 9.57 & \\
\hline$R^{2}$ ajustada & -0.001815 & D.E. Var. Depend. & 0.27 & \\
\hline Error típico regresión & 0.275962 & Criterio Akaike & 0.26 & \\
\hline Suma resid. cuadrado & 41.96136 & Criterio Schwarz & 0.28 & \\
\hline Verosimilitud Log & -71.68763 & Estad.-F & 1.291 & \\
\hline Estad. Durbin-Watson & 0.036338 & Prob(Estad.-F) & 0.99 & \\
\hline
\end{tabular}

Cuadro 6.

El cuadro 6 nos muestra los resultados de la regresión, con los que podemos hacer algunos comentarios.

23 Para que se considere que un estimador es insesgado se debe de cumplir que $E[b]=b$ y que su varianza es $\sigma^{2}[b]=\sigma^{2}\left(X^{T} X\right)^{-1}$. Para que se considere consistente debe cumplirse que la $\operatorname{Prob}[\{b-\beta\}>\delta] \rightarrow 0$ cuando $n \rightarrow \infty$. 
1.- El valor del estadístico $R^{2}$ es cero, esto significa que no existe correlación alguna entre la variable $F(t, T)$ y los residuales.

2.- La variable RES no es estadísticamente significativa para explicar el comportamiento del $F W D$.

3.- El valor de la $\beta_{1}$ es estadísticamente igual a cero, dado que su valor es de $1.99 \mathrm{E}-11$ y su error típico es relativamente alto (.2263), por lo tanto podemos concluir que la covarianza entre la variable $F W D$ y la serie residual es igual cero.

Con el análisis anterior, se puede concluir que se cumple el supuesto 5 de regresión lineal por mínimos cuadrados ordinarios.

\subsection{Supuesto 6: Multicolinealidad}

Se debe entender el concepto de multicolinealidad cuando las variables independientes que se incorporan al modelo de regresión estén altamente correlacionadas. Existen varias formas de observar la multicolinealidad, una de las maneras más tradicionales es verificando el coeficiente de correlación de las variables independientes. Sin embargo, no es factible aplicarlo al modelo analizado, ya que se trata de una regresión con una sola variable independiente. ${ }^{24}$

La multicolinealidad implica que las variables medidas están tan correlacionadas unas con otras, que no es posible analizar con precisión los efectos individuales de cada una de ellas. La multicolinealidad se puede solucionar agregando más datos o realizando otro diseño, porque tal vez el primero fue erróneo. Sin embargo, para los efectos de la hipótesis principal del presente trabajo no aplica el concepto de la multicolinealidad, por lo que se dejará tal cual.

\section{Conclusiones}

Con toda las pruebas realizadas, se cuenta con la evidencia empírica suficiente para poder emitir una opinión fundamentada. Como se puede observar, se han verificado los supuestos básicos de los modelos de regresión múltiple por mínimos cuadrados ordinarios, aplicados al modelo de la Paridad de Tasas de Interés. Los resultados se pueden resumir de la siguiente forma.

Cuadro 7. Resultados.

\begin{tabular}{|c|c|c|}
\hline Supuesto & Cumple & No cumple \\
\hline$\beta$ 's constantes & $\mathrm{X}$ & \\
\hline $\mathrm{E}[\varepsilon]=0$ & $\mathrm{X}$ & \\
\hline $\mathrm{VAR}\left[\varepsilon_{t}\right]=\sigma^{2}$ & & $\mathrm{X}$ \\
\hline $\mathrm{COV}\left[\varepsilon_{1}, \varepsilon_{2}\right]=0$ & $\mathrm{X}$ & \\
\hline $\mathrm{COV}\left[\varepsilon_{t}, X_{1}\right]=0$ & $\mathrm{X}$ & \\
\hline Multicolinealidad & No aplica & \\
\hline$\epsilon_{1} \sim N\left[0, \sigma^{2}\right]$ & & $\mathrm{X}$ \\
\hline
\end{tabular}

24 Existen otros métodos para verificar la existencia de multicolinealidad, tales como las regresiones auxiliares y las estadísticas de Theil. 
Se pudo apreciar de manera clara en la gráfica 5, que el uso de un modelo de regresión lineal fue adecuado para el presente trabajo de investigación. Entonces, se puede concluir que con las pruebas empíricas realizadas, los estimadores obtenidos de la ecuación de regresión son insesgados, pero no son consistentes y por supuesto no son $M E L I{ }^{25}$

Debido a lo mencionado en el párrafo anterior, se concluye que los estimadores obtenidos de la paridad de las tasas de interés para la determinación del tipo de cambio no son eficientes. El problema fundamental que aparece es que la serie del tipo de cambio spot no es estacionaria en la varianza, aunque sí lo es en la media. Este problema ocasiona que los coeficientes de la regresión, $\beta_{0}$ y $\beta_{1}$, no sean estadísticamente iguales a cero y uno respectivamente.

Lo anterior es, a consideración del autor, consecuencia de que después de las elecciones del 2 de julio del 2000, el tipo de cambio se comportó de manera no predecible de conformidad con la teoría de la Paridad de las Tasas de Interés. Otra explicación alternativa a las desviaciones en el nivel de tipo de cambio es que esta teoría funciona bajo esquemas de libre flotación, por lo anterior y tomando en consideración que en México el sistema para la determinación del tipo de cambio no es puramente de libre flotación, se pueden dar comportamientos no predecibles en el mismo. ${ }^{26}$ La recomendación final sería aplicar modelos $G A R C H$ y $A R C H$ para el estudio de los pronósticos de tipo de cambio en el corto plazo, mismos que se dejarán para futuras investigaciones.

\section{Bibliografía}

Amemiya, T. (1985). Advanced Econometrics, Cambridge: Harvard University Press.

Berndt, E. R. (1991). The Practice of Econometrics: Classic and Contemporary, Reading. Alison Wesley

Bhawnani, K. (1997). Forecasting Foreing Exchange Rates in Developing Countries. Applied Economics (29), pp. 51-62.

Brockwell, D. (1996). Introducción Time Series and Forecasting. Springer Texts in Statistics.

Cambell, J., L. Andre, and C. Mackinlay (1997). The Econometrics of Financial Markets. Princeton University Press.

Carstens, A. (1987). Paridad de tasas de interés y riesgo político: Caso México. Estudios Económicos, 2(2), Julio-Diciembre.

Castro y Ortíz, F. (1996). Testing Long-Run Purchasing Power Parity between Asymetric Economics. Department of Economics. The University of Warwick.

Frenkel, J. A. (1996). International Financial Integration: Relations between Interest Rates and Exchange Rates. Dilip Das.

Goldberg, A. S. (1991). A Course in Econometrics, Cambridge, Harvard University Press.

Gouriroux, C. (2001). Financial Econometrics: Problems, Models and Methods. Princeton University Press.

Grabbe, J. O. (1996). International Financial Markets. Prentice Hall.

Greene, W. (1999). Análisis Econométrico. Prentice Hall.

Guerrero, V. M. (1991). Análisis estadístico de series de tiempo económicas. Universidad Autónoma Metropolitana. México.

25 Siglas de "Mejor Estimador Lineal Insesgado", los cuales son aquellos estimadores insesgados y de menor varianza (más eficiente).

26 Esto es debido a que frecuentemente el Banco de México emplea el llamado "corto" o "largo" para regular la oferta monetaria de divisas y para evitar ataques especulativos. Véase Carstens (1987). 
Gujarati, D. (1988). Basic Econometrics. Nueva York, Mc Graw Hill.

Hiroya, A, and T. Waragai (1986). Detecting Structural Changes in the Spot and the Forward Foreing Exchange Rate Market from January 1974 to September 1986. Dilip Das.

Johnston, J. (1984). Econometric Methods. Nueva York, Mc Graw Hill.

Judge, G. G., R. C. Hill, W. E. Griffiths, H. Lutkepohl, and T. Lee (1992). A Guide to Econometrics. Cambridge. MIT Press.

Lyons Richard, K. (1997). A Simultaneos Trade Model of the Foreing Exchange Hot Potato. Journal of International Economics, 42.

Maddala, G. S. (1996). Introducción a la Econometría. Prentice Hall.

Manual de soluciones del paquete econométrico E-views (1996).

Mendenhall, W., D. Wackerly, and R. Sheaffer (1994). Estadística matemática con aplicaciones. Grupo Editorial Iberoamérica.

Mougue, M. and R. Fujihara (1996). International Linkages between Real Short Term Interest Rates. Quarterly Rewiew of Economics and Finance, 36(4).

Notas de clase de Econometría impartida por el Dr. John Dalle Molle, en la Maestría en Finanzas del ITAM, y en las materias de Finanzas Empíricas y Econometría y Análisis Multivariado del Doctorado en Administración, en el Tecnológico de Monterrey, impartidas por el Dr. Carlos Urzúa Macías.

Parker, J. (1991). Exchange Rate Forecasting. The Journal of Business Forecasting, winter, 16-19.

Ross, S. A., R. Westerfield, and J. Jaffe (1996). Corporate Finance. Irwin Mc Graw Hill.

Shapiro, A. (1996). Multinational Financial Management. Prentice Hall.

Werner, A. (1997). Un estudio estadístico sobre el comportamiento de la cotización del peso mexicano frente al dólar y su volatilidad. Documento de Investigación No. 9701. Banco de México.

White, H. (1980). A Heteroskedasticity Consistent Covariance Estimator and a Direct Test for Heteroskedasticity. Econométrica. 